\title{
EDITORIAL
}

\section{The Purpose and Value of Research}

Syed Muhammad Imran Majeed

Human cognition and intellect have always strived for improvement of the human condition. Improvements are primarily based upon development of new knowledge and its application. This remains the prime purpose of all research and its value.

There is often a spatiotemporal disconnect between the occurrence of research and realization of its value. Translation of new knowledge into socio economically valuable application takes time. An apt example is as follow:

Alfred Haar, a Hungarian mathematician-scientist, introduced the idea of HAAR orthogonal system through his doctoral thesis at University of Gottingen, Germany in 1909. Ingrid Daubechics, a Belgian mathematicianphysicist, working at Courount Institute of Mathematical Sciences at New York University, USA, in 1980s developed compactly supported continuous wavelets finding useful application of wavelet theory in digital signal processing especially image compression. Stephen Hallet, originally from France, also helped develop wavelet theory applications in signal and image processing. It took at least seven decades before valuable applications were developed based on Haar's theoretical concepts. New knowledge thus created through research, in one part of the world, may lead to production of valuable applications in a different part of the world, at a different time.

The puritan idea of research as enumerated above, has been corrupted with time. A lot of Ivory tower's research today is taking place to fulfil criteria of job placement and promotions. The concept of impact factor supports this purpose by providing a mechanism, howsoever flawed, of providing a quantitative mechanism to judge research capabilities of individuals and institutions. This lends greater impetus to self-serving research than to society serving. This impact factor approach appears to have helped increase the quantity of research, but certainly not its quality in terms of socio economic value.

Much greater interaction between the elements of quadruple helix (of academia, industry, society and government) is required to improve our understanding to develop means for enhancing the ratio of socioeconomically valuable research to self serving one.

By directing our endeavors to yield better fruits for the society as a whole, serves a far higher purpose and generates much greater value.

\section{Editor-in-Chief}

How to cite this: Majeed SMI. The Purpose and Value of Research. Life and Science. 2021; 2(4): 133-133. doi: http://doi.org/10.37185/LnS.1.1.222

This is an Open Access article distributed under the terms of the Creative Commons Attribution License (http://creativecommons.org/licenses/by/4.0), which permits unrestricted use, distribution, and reproduction in any medium, provided the original work is properly cited. 